\title{
POSITIONAL MODELING IN A SYSTEM WITH TIME DELAY
}

\author{
Marina Blizorukova* \\ Institute of Mathematics and Mechanics, \\ Ural Branch, Russian Academy of Sciences, \\ S. Kovalevskoi str., 16, Ekaterinburg, 620219 Russia \\ msb@imm.uran.ru
}

Abstract The problem of dynamical modeling of unknown control of a system described by equations with time delay is discussed. The constructed algorithm operate synchronically with a process under consideration. It is stable with respect to informational noises and computational errors.

Keywords: modeling, time delay

\section{Introduction. Statement of the problem}

Let us consider a control system described by the vector equation with delay

$$
\begin{gathered}
\dot{x}(t)=f_{1}\left(t, x(t), x\left(t-\alpha_{1}\right), \ldots, x\left(t-\alpha_{k}\right)\right)+ \\
f_{2}\left(t, x(t), x\left(t-\alpha_{1}\right), \ldots, x\left(t-\alpha_{k}\right)\right) u(t), \\
x_{t}(s)=x(t+s)=\varphi(s), \quad s \in[-\alpha, 0], \quad \varphi(\cdot) \in C_{1}[0, \alpha] .
\end{gathered}
$$

Here $t \in T=\left[t_{0}, \vartheta\right], t_{0}<\vartheta$, is time, $x(t) \in R^{n}$ is the system's phase vector, $\alpha_{i}>0, i=1, \ldots, k$ are constant delays, $\left(\alpha=\max \left\{\alpha_{i}: i=\right.\right.$ $1,2, \ldots, k\}), u \in R^{p}$ is a control, $f_{1}(\cdot), f_{2}(\cdot)$ are continuous vector and matrix functions satisfying on $T \times R^{n} \times \ldots \times R^{n}$ the Lipschitz condition:

$$
\begin{gathered}
\left|f_{j}\left(t_{1}, x_{1}, y_{1}^{(1)}, \ldots, y_{k}^{(1)}\right)-f_{j}\left(t_{2}, x_{2}, y_{1}^{(2)}, \ldots, y_{k}^{(2)}\right)\right| \leq \\
c_{j}\left(\left|t_{1}-t_{2}\right|+\left|x_{1}-x_{2}\right|+\sum_{i=1}^{k}\left|y_{i}^{(1)}-y_{i}^{(2)}\right|\right), \quad c_{j} \in(0,+\infty)
\end{gathered}
$$

\footnotetext{
*This work was supported in part by the Russian Foundation for Basic Research (grant \# 01-01-00566).

The original version of this chapter was revised: The copyright line was incorrect. This has been corrected. The Erratum to this chapter is available at DOI: 10.1007/978-0-387-35690-7_44 
for $j=1,2, t_{1}, t_{2} \in T, x_{1}, x_{2}, y_{1}^{(1)}, \ldots, y_{k}^{(1)}, y_{1}^{(2)}, \ldots, y_{k}^{(2)} \in R^{n}$. Throughout the paper we denote by $|\cdot|$ the Euclidean norm in corresponding spaces $R^{n}$ or $R^{n \times p}, C_{1}[0, \alpha]$ is the space of continuously differentiable functions; $x_{t}(s)=x\left(t_{0}+s\right), s \in[-\alpha, 0]$.

Further, any measurable (by Lebesque) function $u(\cdot)$ from a fixed compact $P \subset R^{p}$ is called a control. A solution $x(\cdot)$ of system (1) (in the sense of Caratheodory) with the initial state (2) is called a motion of the system generated by control $u(\cdot)$.

The problem under consideration is in the following. We have the system (1) under the action of some control $u(\cdot), u(t) \in P$ for a.e. $t \in T$. This control $u(\cdot)$ as well as the trajectory $x(\cdot)$ generated by $u(\cdot)$ are unknown. During the process, at frequent enough instances $t=\tau_{i}$ the components of state vector $x\left(\tau_{i}\right)$ are measured. The results of inaccurate measurements are vectors $\xi_{i}$ satisfying the inequalities $\left|\xi_{i}-x\left(\tau_{i}\right)\right| \leq \nu_{i}$, where $\nu_{i}$ is an observation error at the moment $\tau_{i}$. The problem consists in construction of an algorithm that calculates (inaccurately) in real time mode some function $v_{h}(\cdot)$ from the set $U(x(\cdot))$ of controls generating $x(\cdot)$

$$
U(x(\cdot))=\left\{u(\cdot) \in P: x(t)=x\left(t ; t_{0}, x_{t}(\cdot), u(\cdot)\right) \text { a.e. } t \in T\right\}
$$

One of approaches to solving similar problems was presented and developed in [1-3]. For delay systems this method was studied in $[4,5]$. In this work, an algorithm of dynamical reconstruction of an input in a system with delay different from $[4,5]$ is suggested. This algorithm is based on the constructions from [2]. It should be noted that in all papers mentioned above the results of inaccurate measurements satisfy the inequalities $\left|\xi_{i}-x\left(\tau_{i}\right)\right| \leq h, h \in(0,1)$.

\section{The solving algorithm}

We search for the solution of the problem in the class of finite-step dynamical algorithms, i. e. such algorithms that use information only in the nodes of a finite partition and process it between the nodes. As a solution method we choose the method of dynamical reconstruction of controls combined with the well-known in the theory of ill-posed problems discrepancy method [7]. Briefly, the latter consists in the following. Some set containing the sought element is defined on the basis of available inaccurate information. After this, another element, which approximates the one mentioned above (usually, this element is sought as an extremum of a suitable functional) is indicated by some rule.

Let $x_{*}(\cdot)$ be a fixed real motion of the system. We denote by $u_{*}(\cdot)$ the element of the set $U(x(\cdot))$ of minimal $L_{2}(T ; U)$-norm. Let for every $h \in(0,1)$ a uniform partition of the segment $T$ 


$$
\Delta_{h}=\left\{\tau_{h, i}\right\}_{i=0}^{m_{h}}, \quad t_{0}=\tau_{h, 0}<\tau_{h, 1}<\ldots<\tau_{h, m_{h}}=\vartheta, \quad \tau_{h, i}=\tau_{h, i-1}+\delta(h)
$$

with the property

$$
h / \delta(h) \rightarrow 0, \quad \delta(h) \rightarrow 0, \quad \text { as } h \rightarrow 0
$$

be fixed. Let the motion $x(t)$ and the values $\xi_{i}^{h}$ :

$$
\left|\xi_{i}^{h}-x\left(\tau_{h, i}\right)\right| \leq \nu_{i}^{h}
$$

belong to an a priori given compact set $E \subset R^{n}$. We assume that observation errors $\nu_{i}^{h} \geq 0$ satisfy the property

$$
\varphi(h)=\sum_{i=1}^{m_{h}} \nu_{i}^{h} \rightarrow 0 \quad \text { as } \quad h \rightarrow 0 .
$$

Latter condition may be meaningfully treated, for example, in the following sense. The closer is the value $h$ to zero, the better the set of results of measurements of $x(\cdot)$ approximates in average on $T$ this trajectory, i. e.

$$
\sum_{i=0}^{m_{h}} \delta(h)\left|\xi_{i}^{h}-x\left(\tau_{h, i}\right)\right| \leq \delta(h) \varphi(h) \rightarrow 0 \quad \text { as } \quad h \rightarrow 0 .
$$

Introduce constants $c_{0}>0, c_{u}>0, c_{*}>0$ such that

$$
\begin{gathered}
\left|f_{1}\left(t, x, y_{1}, \ldots, y_{k}\right)+f_{2}\left(t, x, y_{1}, \ldots, y_{k}\right) u\right| \leq c_{0} \quad \forall t \in T \\
x, y_{i} \in E, i=1,2, \ldots, k, \quad u \in P ; \quad|u| \leq c_{u} \quad \forall u \in P \\
\left|\varphi\left(t_{1}\right)-\varphi\left(t_{2}\right)\right| \leq c_{0}\left|t_{1}-t_{2}\right| \quad \forall t \in[0, \alpha] ; \\
\left|x\left(\tau_{i}\right)\right| \leq c_{*}, \quad\left|\xi_{i}^{h}\right| \leq c_{*}, \quad \forall i \in\left[0: m_{h}\right], \quad h \in(0,1) .
\end{gathered}
$$

Let us turn to the description of the algorithm. We choose an auxiliary discrete control system (a model) corresponding to system (1).

The model state at every moment $\tau_{h, i}$ is characterized by the vector

$$
\begin{gathered}
z^{h}\left(\tau_{h, i+1}\right)=z^{h}\left(\tau_{h, i}\right)+ \\
{\left[f_{1}\left(\tau_{h, i-1}, \xi_{i-1}^{h}, \xi_{h}\left(\tau_{h, i-1}-\alpha_{1}\right), \ldots, \xi_{h}\left(\tau_{h, i-1}-\alpha_{k}\right)\right)+\right.} \\
\left.f_{2}\left(\tau_{h, i-1}, \xi_{i-1}^{h}, \xi_{h}\left(\tau_{h, i-1}-\alpha_{1}\right), \ldots, \xi_{h}\left(\tau_{h, i-1}-\alpha_{k}\right)\right) v_{h}\left(\tau_{h, i}\right)\right] \delta \\
z_{h}\left(\tau_{h, 1}\right)=\varphi(0),
\end{gathered}
$$

where

$$
\begin{aligned}
& \xi_{h}\left(\tau_{h, i}-\alpha_{j}\right)=\xi_{i-m_{h}^{0}(j)}^{h} \\
& \vartheta_{m_{h}(j)}=\alpha_{j}-m_{h}(j) \delta(h), \quad m_{h}^{0}(j)= \begin{cases}m_{h}(j), & \vartheta_{m_{h}(j)}=0, \\
m_{h}(j)+1, & \vartheta_{m_{h}(j)} \neq 0 .\end{cases}
\end{aligned}
$$


The symbol $m_{h}(j)$ denotes the integral part of the number $\alpha_{j} / \delta(h)$.

A model control is chosen according to the principle of extremal shift [6]. We suppose that at every moment $\tau_{h, i}(i \geq 1)$ the closed set

$$
\begin{gathered}
\Omega_{h, i}=\Omega_{h, i}\left(\xi_{i}^{h}, \xi_{i-1}^{h}\right)=\left\{v \in P:\left(z_{h}\left(\tau_{h, i}\right)-\xi_{i-1}^{h}\right)^{\prime} \times\right. \\
{\left[f_{1}\left(\tau_{h, i-1}, \xi_{i-1}^{h}, \xi_{h}\left(\tau_{h, i-1}-\alpha_{1}\right), \ldots, \xi_{h}\left(\tau_{h, i-1}-\alpha_{k}\right)\right)+\right.} \\
\left.f_{2}\left(\tau_{h, i-1}, \xi_{i-1}^{h}, \xi_{h}\left(\tau_{h, i-1}-\alpha_{1}\right), \ldots, \xi_{h}\left(\tau_{h, i-1}-\alpha_{k}\right)\right) v\right]- \\
\left.\left(z_{h}\left(\tau_{h, i}\right)-\xi_{i}^{h}\right)^{\prime} \frac{\xi_{i}^{h}-\xi_{i-1}^{h}}{\delta} \leq \sigma_{h, i}^{\delta}\right\}
\end{gathered}
$$

is constructed on the basis of measurement results $\xi_{i}^{h}$ and $\xi_{i-1}^{h}$. Here the prime stands for transposition, the values $\sigma_{h, i}^{\delta}$ being of the form

$$
\begin{gathered}
\sigma_{h, i}^{\delta}=c_{0}^{2} \delta+c_{0}\left(\nu_{i}^{h}+\nu_{i-1}^{h}\right)+\left(2 c_{*}+\nu_{i-1}^{h}\right)\left(c_{1}+c_{2} c_{u}\right)\left[c_{0}(k+2) \delta+\nu_{i-1}^{h}+\right. \\
\sum_{j=1}^{k}\left(\nu_{i-m_{h}(j)}^{h}+\nu_{i-1-m_{h}(j)}^{h}\right)+\frac{2 c_{*}}{\delta}\left(\nu_{i}^{h}+\nu_{i-1}^{h}\right) .
\end{gathered}
$$

$\nu_{i-m_{h}(j)}^{h}=0$ for $i-m_{h}(j)<0$. Following the ideology of discrepancy method, we indicate the law of forming the control in the model:

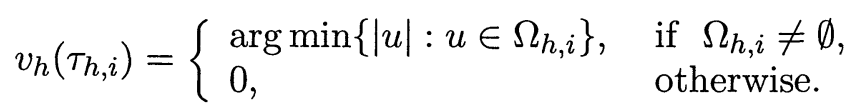

Introduce the family of functions

$$
v_{h}(t)=v_{h}\left(\tau_{h, i}\right) \quad \text { for } \quad t \in\left[\tau_{h, i}, \tau_{h, i+1}\right), \quad i \in\left[0, m_{h}-1\right] .
$$

The following theorem is true.

Theorem 1 A family of controls $\left\{v_{h}(\cdot)\right\}, h \in(0,1)$, defined by (4)-(8), satisfies the following property

$$
\left|v_{h}(\cdot)-u_{*}(\cdot)\right|_{L_{2}(T ; U)} \rightarrow 0 \quad \text { as } h \rightarrow 0 .
$$

The proof of this theorem is based on the following auxiliary statement.

We introduce a value $\varepsilon_{j+1}=\left|z_{j+1}-x_{j}\right|^{2}, \quad x_{j}=x_{*}\left(\tau_{j}\right), \quad z_{j}=z_{h}\left(\tau_{j}\right)$.

Lemma 1 The inequality

$$
\varepsilon_{j} \leq 2 \delta(h) \sum_{i=0}^{j} \sigma_{n, i}^{\delta}+6 c_{0} \delta\left(\vartheta-t_{0}\right)+4\left(c_{*}+c_{0}\right) \sum_{i=0}^{j} \nu_{i}^{h}
$$

is true.

Proof of Lemma 1. For $\vartheta_{m_{h}(j)}=0$, we get

$$
\left|x\left(t-\tau_{j}\right)-\xi_{i-1, j}^{h}\right|_{n} \leq c_{0} \delta+\left(\nu_{i-m_{h}(j)}^{h}+\nu_{i-1-m_{h}(j)}^{h}\right), \quad j \in[1: k],
$$


where $t \in\left[t_{i-1}, t_{i}\right]$. If $\vartheta_{m_{h}(j)} \neq 0$, then $\left|x(t)-\xi_{i-1}^{h}\right|_{n} \leq c_{0} \delta+\nu_{i-1}^{h}$. Therefore,

$$
\begin{gathered}
\left|x(t)-\xi_{i-1}^{h}\right|_{n}+\left|x\left(t-\tau_{1}\right)-\xi_{i-1,1}^{h}\right|_{n}+\ldots+\left|x\left(t-\tau_{k}\right)-\xi_{i-1, k}^{h}\right|_{n} \leq \\
c_{0} \delta(k+1)+\nu_{i-1}^{h}+\sum_{j=1}^{k}\left(\nu_{i-m_{h}(j)}^{h}+\nu_{i-1-m_{h}(j)}^{h}\right) .
\end{gathered}
$$

It is easy to see that (for $c_{0} \geq 1$ )

$$
\left|\Delta f_{1}\right|_{n} \leq c_{1}\left(\delta c_{0}(k+2)+\nu_{i-1}^{h}+\sum_{j=1}^{k}\left(\nu_{i-m_{h}(j)}^{h}+\nu_{i-1-m_{h}(j)}^{h}\right)\right) .
$$

We obtain analogously

$$
\left|\Delta f_{2}\right|_{n} \leq c_{u} c_{2}\left(\delta c_{0}(k+2)+\nu_{i-1}^{h}+\sum_{j=1}^{k}\left(\nu_{i-m_{h}(j)}^{h}+\nu_{i-1-m_{h}(j)}^{h}\right)\right) .
$$

Hence,

$$
\begin{gathered}
|\Delta f|_{n} \leq \mu(\delta, i)=\left(c_{1}+c_{2} c_{u}\right) c_{0}(k+2) \delta+ \\
+\left(c_{1}+c_{2} c_{u}\right)\left(\nu_{i-1}^{h}+\sum_{j=1}^{k}\left(\nu_{i-m_{h}(j)}^{h}+\nu_{i-1-m_{h}(j)}^{h}\right)\right) .
\end{gathered}
$$

Taking into account the following inequalities

$$
\begin{aligned}
\left|z^{h}\left(t_{i}\right)-\xi_{i-1}^{h}\right|_{n} & \leq\left|z^{h}\left(t_{i}\right)\right|_{n}+\left|\xi_{i-1}^{h}-x\left(t_{i-1}\right)\right|_{n}+\left|x\left(t_{i-1}\right)\right|_{n} \leq \\
& \leq 2 c_{*}+\nu_{i-1}^{h} \\
\left|\xi_{i}^{h}-\xi_{i-1}^{h}\right|_{n} & \leq\left|\xi_{i}^{h}-x\left(t_{i}\right)\right|_{n}+\left|\xi_{i-1}^{h}-x\left(t_{i-1}\right)\right|_{n}+\mid x\left(t_{i}\right)- \\
& -\left.x\left(t_{i-1}\right)\right|_{n} \leq c_{0} \delta+\nu_{i}^{h}+\nu_{i-1}^{h},
\end{aligned}
$$

we have

$$
\begin{aligned}
\mu_{i}^{(2)}= & \left(\xi_{i}^{h}-\xi_{i-1}^{h}, \int_{t_{i-1}}^{t_{i}}\left[f_{1}\left(t, x(t), x\left(t-\tau_{1}\right), \ldots, x\left(t-\tau_{k}\right)\right)+\right.\right. \\
& \left.\left.f_{2}\left(t, x(t), x\left(t-\tau_{1}\right), \ldots, x\left(t-\tau_{k}\right)\right) u_{*}(t)\right] d t\right)_{n} \leq \\
& \left|\xi_{i}^{h}-\xi_{i-1}^{h}\right|_{n} \int_{t_{i-1}}^{t_{i}} c_{0} d t \leq c_{0} \delta\left(c_{0} \delta+\nu_{i}^{h}+\nu_{i-1}^{h}\right) .
\end{aligned}
$$

From (10), (11) we also deduce

$$
\mu_{i}^{(1)} \leq\left|z^{h}\left(t_{i}\right)-\xi_{i-1}^{h}\right|_{n} \delta|\Delta f|_{n} \leq\left(2 c_{*}+h_{i-1}\right) \delta \mu(\delta, i) .
$$


This and the assumption that $\delta \in(0,1), h \in(0,1), h / \delta \in(0,1)$ imply

$$
\mu_{i} \leq \rho_{\delta}(i) \delta
$$

where $\rho_{\delta}(i)=c_{0}\left(c_{0} \delta+\nu_{i}^{h}+\nu_{i-1}^{h}\right)+\left(2 c_{*}+\nu_{i-1}^{h}\right) \mu(\delta, i)$.

Using (11), (12), we get

$$
\begin{gathered}
\left(z^{h}\left(t_{i}\right)-\xi_{i-1}^{h}, f_{1}\left(t_{i-1}, \xi_{i-1}^{h}, \xi_{i-1,1}^{h}, \ldots, \xi_{i-1, k}^{h}\right)+\right. \\
\left.f_{2}\left(t_{i-1}, \xi_{i-1}^{h}, \xi_{i-1,1}^{h}, \ldots, \xi_{i-1, k}^{h}\right) \delta^{-1} \int_{t_{i-1}}^{t_{i}} u_{*}(\tau) d \tau\right)_{n}- \\
\left(z^{h}\left(t_{i}\right)-\xi_{i}^{h}, \xi_{i}^{h}-\xi_{i-1}^{h}\right)_{n} \delta^{-1} \leq \rho_{\delta}(i)+\left(2 c_{*}+\nu_{i-1}^{h}\right)\left(\nu_{i}^{h}+\nu_{i-1}^{h}\right) \delta^{-1} .
\end{gathered}
$$

Further,

$$
\begin{gathered}
\varepsilon_{j+1} \leq \varepsilon_{j}+2\left(z_{j}-\xi_{j-1}^{h},\left[f_{1}\left(t_{j-1}, \xi_{j-1}^{h}, \xi_{j-1,1}^{h}, \ldots, \xi_{j-1, k}^{h}\right)+\right.\right. \\
\left.\left.f_{2}\left(t_{j-1}, \xi_{j-1}^{h}, \xi_{j-1,1}^{h}, \ldots, \xi_{j-1, k}^{h}\right) v^{h}\left(t_{j}\right)\right]\right)_{n} \delta+2 \nu_{j-1}^{h} c_{0} \delta+ \\
2\left(z_{j}-x_{j}, x_{j}-x_{j-1}\right)_{n}+4\left(c_{0} \delta\right)^{2}+2\left|x_{j}-x_{j-1}\right|_{n}^{2} .
\end{gathered}
$$

Hence,

$$
\begin{gathered}
\varepsilon_{j+1} \leq \varepsilon_{j}+2\left(z_{j}-\xi_{j-1}^{h},\left[f_{1}\left(t_{j-1}, \xi_{j-1}^{h}, \xi_{j-1,1}^{h}, \ldots, \xi_{j-1, k}^{h}\right)+\right.\right. \\
\left.\left.f_{2}\left(t_{j-1}, \xi_{j-1}^{h}, \xi_{j-1,1}^{h}, \ldots, \xi_{j-1, k}^{h}\right) v^{h}\left(t_{j}\right)\right]\right)_{n} \delta+ \\
2\left(z_{j}-\xi_{j}^{h}, x_{j}-x_{j+1}\right)_{n}+6\left(c_{0} \delta\right)^{2}+2 c_{0} \delta\left(\nu_{j}^{h}+\nu_{j-1}^{h}\right) .
\end{gathered}
$$

Besides,

$$
\begin{gathered}
\left|\left(z_{j}-\xi_{j}^{h}, x_{j}-x_{j-1}\right)_{n}-\left(z_{j}-\xi_{j}^{h}, \xi_{j}^{h}-\xi_{j-1}^{h}\right)_{n}\right| \leq \\
\left(\nu_{j}^{h}+\nu_{j-1}^{h}\right)\left|z_{j}-\xi_{j}^{h}\right|_{n} \leq 2 c_{*}\left(\nu_{j}^{h}+\nu_{j-1}^{h}\right) .
\end{gathered}
$$

Therefore

$$
\varepsilon_{j+1} \leq \varepsilon_{j}+2 \sigma_{h, j}^{\delta} \delta+2\left(c_{*}+c_{0} \delta\right)\left(\nu_{j}^{h}+\nu_{j-1}^{h}\right)+6\left(c_{0} \delta\right)^{2} .
$$

Finally, we have

$$
\varepsilon_{j+1} \leq 2 \delta \sum_{i=0}^{j} \sigma_{h, i}^{\delta}+6 c_{0}^{2} \delta\left(\vartheta-t_{0}\right)+2\left(c_{*}+c_{0} \delta\right) \sum_{i=1}^{j}\left(\nu_{j}^{h}+\nu_{j-1}^{h}\right) .
$$

This finishes the proof of the lemma.

Remark In the case when the set $P$ is a ball:

$$
P=\left\{u \in R^{N}:|u|_{N} \leq a\right\},
$$

the control in the model can be written explicitly: 


$$
v_{i}^{h}= \begin{cases}0, & \text { if } \quad b_{i}^{h}=0 \text { or } \chi_{i}^{h}>0 \\ \chi_{i}^{h} \frac{b_{i}^{h}}{\left|b_{i}^{h}\right|_{n}^{2}}, & \text { if } \quad b_{i}^{h} \neq 0 \text { and } \chi_{i}^{h} \leq 0 .\end{cases}
$$

Here

$$
\begin{gathered}
b_{i}^{h}=\left(z_{i}-\xi_{i-1}^{h}, f_{2}\left(t_{i-1}, \xi_{i-1}^{h}, \xi_{i-1,1}^{h} \ldots, \xi_{i-1, k}^{h}\right)\right)_{n} \\
\chi_{i}^{h}=\sigma_{h}^{\delta}+\left(z_{i}-\xi_{i-1}^{h}, \frac{\xi_{i}^{h}-\xi_{i-1}^{h}}{\delta}-f_{1}\left(t_{i-1}, \xi_{i-1}^{h}, \xi_{i-1,1}^{h} \ldots, \xi_{i-1, k}^{h}\right)\right)_{n} .
\end{gathered}
$$

\section{Conclusion}

The problem of dynamical modeling (reconstruction) of an unknown input that determines the motion of a dynamical system with time delay through given inaccurate measurements of the system's current state is considered. The scheme of solving this problem leans upon the method of auxiliary positionally controlled models. The suggested solution method consists in the combination of the method of dynamical reconstruction of controls and a dynamical modification of the well-known in the theory of ill-posed problems discrepancy method. A dynamical algorithm working "in real time" based on this method is discussed.

\section{References}

[1] Kryazimskii, A. V. and Osipov, Yu. S. (1983). Modelling of a control in a dynamic system. Engineering Cybernetics, 21:38-47. in Russian.

[2] Kryazimskii, A. V. and Osipov, Yu. S. (1988). On methods of positional modeling of a control in a dynamic system. 34-44. Qualitative questions of the theory of differential equations and control systems, Urals. Sci. Center, Sverdlovsk. in Russian.

[3] Kryazimskii, A. V. and Osipov, Yu. S. (1995). Inverse Problems for Ordinary Differential Equations: Dynamical Solutions. Gordon and Breach. London.

[4] Kryazimskii, A. V., Maksimov, V. I. and Osipov, Yu. S. (1983). On positional modelling in dynamic systems. Prikl. math. mech., 47:883-889.

[5] Blizorukova, M.S. and Maksimov, V.I. (1999). On the reconstruction of a pair "control-trajectory" in a system with hereditary. Problems of control and informatics, 4:37-48. in Russian.

[6] Krasovskii, N.N. and Subbotin, A.I. (1988). Game-Theoretical Control Problems. Springer Verlag. New York - Berlin.

[7] Tikhonov, A.N. and Arsenin, V.Ya. (1977). Solution of Ill-posed Problems. John Wiley, New York. 